\title{
A self-configured link adaptation for green LTE downlink transmission
}

\begin{abstract}
Green deployment for cellular eNodeBs has been proposed recently to save power and reduce the huge amount of carbon dioxide (CO2) emitted by traditional power-hungry base stations. Green eNodeBs should also be subjected to restrictions on high data rate and quality of service (QoS), which both entail a high level of power consumption. In this regard, this paper addresses the trade-off between energy efficiency (EE) and spectral efficiency (SE) in both traditional and green long-term evolution eNodeBs without sacrificing the QoS. EE is proved to monotonically increase with SE in traditional macrocells and quasi-concave in green macrocells. Accordingly, a new mapping between channel quality indicator and modulation and coding scheme is proposed to address EEÏ SE trade-off with the use of a multi-criteria decision-making technique. Then, a self-configured link adaptation (SCLA) algorithm is developed to ensure that the priority weights related to EE and SE are adapted according to network load with the use of real-time cross-layer optimization. Simulation results show that the proposed SCLA provides a significant gain in EE and 52\% reduction of $\mathrm{CO} 2$ while maintaining SE close to the optimal value. Current and next-generation cellular networks require such interactive techniques in order to be self-optimised without complex modifications.
\end{abstract}

Keyword: Energy efficiency; Spectral efficiency; eNodeBs 